\title{
PERFIL ISOENZIMÁTICO DE MAÍCES NATIVOS DEL ISTMO DE TEHUANTEPEC, OAXACA, MÉXICO. I. CARACTERIZACIÓN DE GRUPOS
}

\author{
ISOZYMATIC PROFILE OF MAIZES NATIVE TO THE TEHUANTEPEC ISTHMUS, OAXACA, \\ MÉXICO. I. CHARACTERIZATION OF GROUPS
}

\author{
Gustavo López Romero, Amalio Santacruz Varela*, Abel Muñoz Orozco, Fernando Castillo González, \\ Leobigildo Córdova Téllez y Humberto Vaquera Huerta ${ }^{1}$
}

${ }^{1}$ Colegio de Postgraduados, Campus Montecillo. km 36.5 Carretera México-Texcoco. 56230, Montecillo, Texcoco, Edo. de México. Tel. 01-595-9520200 Ext. 1570, Fax 01-595-9520262.

* Autor para correspondencia (asvarela@colpos.mx)

\section{RESUMEN}

El maíz (Zea mays L. ssp. mays) es la especie cultivada más importante y diversa en México. La diversidad genética de las principales razas mexicanas de maíz se ha estudiado con base en datos agronómicos, morfológicos y marcadores moleculares como isoenzimas, aunque en este último caso solamente se ha considerado un número escaso de poblaciones representativas de cada raza de maíz. Por tanto, faltan estudios que revelen con profundidad el grado de variación genética existente dentro de razas. El objeto de esta investigación fue evaluar el polimorfismo isoenzimático de poblaciones de maíz del Istmo de Tehuantepec, Oaxaca, México, área de distribución de la raza Zapalote Chico. Se evaluaron 40 poblaciones nativas y 10 representativas de otras razas como referencia para analizar la variación genética de 19 loci isoenzimáticos. Se encontró un total de 52 alelos con un promedio de 2.7 alelos por locus. Las poblaciones nativas tuvieron 49 alelos, que supera en 10 alelos a las poblaciones representativas de otras razas. El análisis de la diversidad genética de las poblaciones nativas, basado en el estadístico de diferenciación genética (Gst), mostró que $88 \%$ de la variación isoenzimática reside dentro de poblaciones y $12 \%$ entre poblaciones. El análisis de conglomerados separó dos grupos de maíces nativos, el primero integró las poblaciones de maíz de la raza Zapalote Chico, mientras que el segundo agrupó las poblaciones conocidas localmente como Maíz Grande, con influencia de las razas Zapalote Chico e introgresión de Tuxpeño, Vandeño y Tepecintle. El análisis de las frecuencias génicas reveló el perfil isoenzimático de cada uno de los agrupamientos detectados. Los resultados obtenidos indican la presencia de variación genética en poblaciones de maíz nativas de Istmo de Tehuantepec, que puede aprovecharse en programas de mejoramiento genético.

Palabras clave: Zea mays, Zapalote Chico, diversidad genética, polimorfismo, isoenzimas.

\section{SUMMARY}

Maize (Zea mays L. ssp. mays) is the most important and diverse of the cultivated species in México. Genetic diversity of the primary Mexican races of maize has been studied on the basis of agronomic, morphological and molecular markers such as isozymes data; howe- ver, the studies involving isozymes have only considered a low number of representative populations for each race; therefore, more in-depth studies are necessary for revealing the extent of genetic variation within races. The objective of this research was to evaluate the isozymatic polymorphism of maize populations from the Tehuantepec Isthmus, at Oaxaca, México, which is the area of natural distribution of the Zapalote Chico race. Forty native populationns and 10 populations representative of other maize races, were evaluated in order to analyze genetic variation of 19 isozyme loci. A total of 52 alleles, with an average of 2.7 alleles per locus, were found. Native populations had 49 alleles, outnumbering representative populations of other races by 10 alleles. Analysis of genetic diversity of the native populations, based on the statistic for genetic differentiation (GsT) showed that $88 \%$ of the isozymatic variation resides within populations and $12 \%$ among populations. Cluster analysis separated two groups of native maize, the first one integrated populations of the Zapalote Chico race, while the second one grouped populations locally known as Maíz Grande, with some influence of races Zapalote Chico and introgression of Tuxpeño, Vandeño and Tepecintle. Gene frequencies analysis revealed the isozymatic profile for each group. Results indicate the presence of genetic variation into native maize populations from the Tehuantepec Isthmus, readily available for use in breeding programs.

Index words: Zea mays, Zapalote Chico, genetic diversity, polymorphism, isozymes.

\section{INTRODUCCIÓN}

La diversidad genética constituye la base para la evolución de los organismos, y en la agricultura tal diversidad constituye el reservorio de genes para hacer frente a una amplia gama de factores adversos e incrementar los rendimientos de las cosechas.

El maíz (Zea mays L.) es la especie cultivada con la mayor diversidad genética en el ámbito mundial. Para México se han descrito al menos 59 razas (Sánchez et al., 2000), en las cuales se ha estudiado la diversidad mediante 
caracteres morfológicos y alelos isoenzimáticos (Doebley et al., 1985, Sánchez et al., 2000); sin embargo, es notoria la falta de estudios de diversidad dentro de razas o dentro de regiones agrícolas específicas.

En el Istmo de Tehuantepec el maíz nativo o criollo es un cultivo importante. En el año 2008 se sembraron en la región 84066 hectáreas con maíz, $87 \%$ en condiciones de temporal y $13 \%$ en riego, con un rendimiento promedio de 1.4 y $1.7 \mathrm{t} \mathrm{ha}^{-1}$, respectivamente (SIAP, 2009). Los indígenas Zapotecas habitan principalmente en la zona de la planicie costera, donde predomina el cultivo del maíz de la raza Zapalote Chico y prevalecen las condiciones más extremas de viento en invierno, y con calor y desecación en verano. En las localidades de la zona montañosa las condiciones ambientales son contrastantes a las de la planicie, pues las temperaturas son más frescas y los vientos menos intensos; ahí las poblaciones de maíz son más tardías, con influencia del complejo de razas dentadas tropicales como Vandeño, Tuxpeño y Tepecintle.

Son pocos los estudios publicados en la raza Zapalote Chico, entre ellos el realizado por Ramírez et al. (1988) sobre selección familial, quienes al no detectar respuesta a la selección concluyeron que su variabilidad era estrecha, y desde entonces prevalece esa idea entre los fitomejoradores. Sin embargo, en una exploración posterior más amplia, López et al. (2005) identificaron a nivel fenotípico una variabilidad importante, que ahora se pretende corroborar con marcadores bioquímicos, con el objeto de posicionar a esta raza en un escenario más favorable para su aprovechamiento en los programas de mejoramiento genético, lo que hasta ahora no ha ocurrido, a pesar de que cuenta con una serie de atributos sobre calidad de grano y resistencia a factores adversos. Los objetivos en esta investigación fueron: a) valorar el nivel de polimorfismo de isoenzimas en poblaciones de maíz de la región del Istmo de Tehuantepec, Oaxaca, y b) clasificar las poblaciones en grupos con base en la variación isoenzimática.

\section{MATERIALES Y MÉTODOS}

\section{Descripción del área de estudio}

La región del Istmo de Tehuantepec, Oaxaca, México comprende los distritos de Tehuantepec y Juchitán, los cuales integran 54 municipios. Ahí convergen dos sistemas montañosos; al oriente, la Sierra Madre de Chiapas, y al poniente, la Sierra Madre de Oaxaca, cuya conformación altitudinal permite el paso de fuertes corrientes de viento provenientes del norte durante los meses de octubre a marzo, con velocidades que superan los $100 \mathrm{~km} \mathrm{~h}^{-1}$. En esta región la altitud varía de cero a $2250 \mathrm{~m}$; la temperatura promedio anual de 20 a $26{ }^{\circ} \mathrm{C}$; la precipitación oscila entre 550 y $1490 \mathrm{~mm}$, y la evaporación fluctúa entre 1200 y $2600 \mathrm{~mm}$ anuales. Lo anterior define tres tipos climáticos: cálido semiárido, al poniente de la región; cálido subhúmedo, predominante en la región, y semicálido subhúmedo, en las áreas con mayor altitud.

\section{Material genético}

Se evaluaron 40 poblaciones representativas del patrón morfológico de 120 poblaciones nativas de 38 municipios del Istmo de Tehuantepec, Oaxaca (López et al., 2005), incluyendo poblaciones de Zapalote Chico y Maíz Grande. Es importante señalar que los agricultores del Istmo de Tehuantepec usan el término Maíz Chico para referirse a poblaciones de la raza Zapalote Chico, típica de esa región y el término Maíz Grande para referirse al maíz diferente de Zapalote Chico. El término Maíz Grande no se refiere a la raza Zapalote Grande y no corresponde en forma pura a ninguna de las razas que identifican los fitomejoradores; su estrato más precoz, que se cultiva más cerca de la planicie costera tiene similitud, con Zapalote Chico, mientras que los estratos más tardíos están más alejados de la costa, conservan parentesco con Zapalote Chico pero con introgresión de otras razas como Vandeño, Tuxpeño y Tepecintle. En este contexto, el Maíz Grande posiblemente justifique su identificación como un complejo independiente, requiriéndose para ello de estudios adicionales.

Los datos de ubicación geográfica y sitios de colecta se muestran en los Cuadros 1 y 2 . Adicionalmente, se evaluaron 10 poblaciones representativas de otras razas: LADA-434 (Nal-Tel) proporcionada por el banco de germoplasma de la Universidad Autónoma Chapingo, más las poblaciones CHIS-224 (Zapalote Grande), CHIS-578 (Clavillo), GUER-96 (Vandeño), OAXA-54 (Zapalote Chico), OAXA-244 (Olotillo), YUCA-108 (Nal-Tel), YUCA-117 (Dzit-Bacal), VERA-39 (Tuxpeño) y VERA168 (Dzit-Bacal), proporcionadas por el Centro Internacional de Mejoramiento de Maíz y Trigo (CIMMYT) (DBMU, 1992).

\section{Análisis isoenzimático}

Se analizaron 19 loci pertenecientes a las enzimas: fosfatasa ácida (Acp1 y Acp4), alcohol deshidrogenasa $($ Adh1), catalasa (Cat3), esterasa (E8), $\beta$-glucosidasa (Glu1), glutamato oxaloacetato transaminasa (Got1, Got2 y Got3), isocitrato deshidrogenasa (Idh1, Idh2), malato deshidrogenasa (Mdh1, Mdh2, Mdh3, Mdh4 y Mdh5), 6fosfogluconato deshidrogenasa (Pgd1 y Pgd2) y fosfato isomerasa (Phi1), por contar con protocolos de tinción 
confiables y estar distribuidos en ocho de los 10 cromosomas del maíz (Stuber et al., 1988). Las enzimas se extrajeron a partir de coleoptilos de 10 plántulas de $6 \mathrm{~d}$ después de siembra en cada población y de individuos de las líneas B73, Mo17, Tx303 y Mo24W, que son utilizadas como testigos con alelos conocidos. Posteriormente, las enzimas se separaron mediante electroforesis en geles de almidón, y las bandas se revelaron con los protocolos descritos por Stuber et al. (1988). Después de la tinción se tomaron fotografías de los zimogramas para su lectura, y se tomó como referencia el desplazamiento de los alelos conocidos de las líneas testigo y el patrón de bandeo con las guías informadas por Stuber et al. (1988).

Cuadro 1. Sitio de colecta de las poblaciones de maíz Zapalote Chico utilizadas en el estudio.

\begin{tabular}{|c|c|c|c|c|}
\hline Población & Lugar de colecta & $\begin{array}{l}\text { Altitud } \\
\text { (m) }\end{array}$ & $\begin{array}{l}\text { Latitud } \\
\text { Norte }\end{array}$ & $\begin{array}{l}\text { Longitud } \\
\text { Oeste }\end{array}$ \\
\hline 3 & Jalapa del Marqués, Oax. & 140 & $16^{\circ} 26^{\prime} 42^{\prime \prime}$ & $95^{\circ} 27^{\prime} 03^{\prime \prime}$ \\
\hline 5 & Chihuitán, Oax. & 109 & $16^{\circ} 35^{\prime} 34^{\prime \prime}$ & $95^{\circ} 10^{\prime} 02^{\prime \prime}$ \\
\hline 10 & Tehuantepec, Oax. & 35 & $16^{\circ} 22^{\prime} 48^{\prime \prime}$ & $95^{\circ} 13^{\prime} 44^{\prime \prime}$ \\
\hline 11 & Unión Hidalgo, Oax. & 12 & $16^{\circ} 28^{\prime} 15^{\prime}$, & $95^{\circ} 50^{\prime} 00^{\prime \prime}$ \\
\hline 12 & San Mateo del Mar, Oax. & 5 & $16^{\circ} 12^{\prime} 33^{\prime \prime}$ & $94^{\circ} 59^{\prime} 01^{\prime \prime}$ \\
\hline 13 & San Blas Atempa, Oax. & 22 & $16^{\circ} 17^{\prime} 12^{\prime \prime}$ & $95^{\circ} 10^{\prime} 25^{\prime \prime}$ \\
\hline 14 & Huanacastal, Oax. & 30 & $16^{\circ} 28^{\prime} 37^{\prime \prime}$ & $94^{\circ} 28^{\prime} 06^{\prime \prime}$ \\
\hline 17 & Santa Maria Guienagati, Oax. & 350 & $16^{\circ} 44^{\prime} 15^{\prime}$, & $95^{\circ} 19^{\prime} 03^{\prime \prime}$ \\
\hline 18 & Santa Maria Guienagati, Oax. & 350 & $16^{\circ} 44^{\prime} 15^{\prime}$, & $95^{\circ} 21^{\prime} 14^{\prime \prime}$ \\
\hline 21 & Mixtequilla, Oax. & 40 & $16^{\circ} 22^{\prime} 54^{\prime \prime}$ & $95^{\circ} 15^{\prime} 27^{\prime \prime}$ \\
\hline 22 & Reforma de Pineda, Oax. & 27 & $16^{\circ} 23^{\prime} 47^{\prime}$, & $94^{\circ} 27^{\prime} 37^{\prime \prime}$ \\
\hline 25 & San Pedro Huilotepec, Oax. & 20 & $16^{\circ} 14^{\prime} 38^{\prime \prime}$ & $95^{\circ} 09^{\prime} 08^{\prime \prime}$ \\
\hline 26 & Santo Domingo Ingenio, Oax. & 58 & $16^{\circ} 35^{\prime} 06^{\prime \prime}$ & $94^{\circ} 45^{\prime} 57^{\prime}$, \\
\hline 27 & Chahuites, Oax. & 29 & $16^{\circ} 17^{\prime} 18^{\prime \prime}$ & $94^{\circ} 11^{\prime} 26^{\prime \prime}$ \\
\hline 29 & Santa María Totolapilla, Oax. & 500 & $16^{\circ} 35^{\prime} 58^{\prime \prime}$ & $95^{\circ} 37^{\prime} 25^{\prime \prime}$ \\
\hline 30 & Santa Maria Totolapilla, Oax. & 500 & $16^{\circ} 35^{\prime} 58^{\prime}$, & $95^{\circ} 37^{\prime} 25^{\prime}$, \\
\hline 35 & Tapanatepec, Oax. & 20 & $16^{\circ} 21^{\prime} 46^{\prime \prime}$ & $94^{\circ} 11^{\prime} 30^{\prime \prime}$ \\
\hline 36 & San Francisco Ixhuatán, Oax. & 17 & $16^{\circ} 21^{\prime} 00^{\prime \prime}$ & $94^{\circ} 28^{\prime} 57^{\prime \prime}$ \\
\hline 37 & Jalapa del Marqués, Oax. & 177 & $16^{\circ} 26^{\prime} 22^{\prime \prime}$ & $95^{\circ} 30^{\prime} 37^{\prime \prime}$ \\
\hline 38 & Santo Domingo Ingenio, Oax. & 58 & $16^{\circ} 35^{\prime} 06^{\prime \prime}$ & $94^{\circ} 45^{\prime} 57^{\prime \prime}$ \\
\hline 39 & Chahuites, Oax. & 29 & $16^{\circ} 17^{\prime} 18^{\prime \prime}$ & $94^{\circ} 11^{\prime} 26^{\prime \prime}$ \\
\hline 40 & Santiago Laollaga, Oax. & 130 & $16^{\circ} 35^{\prime} 01^{\prime \prime}$ & $95^{\circ} 12^{\prime} 37^{\prime \prime}$ \\
\hline \multicolumn{5}{|c|}{ Cuadro 2. Sitio de colecta de las poblaciones de Maíz Grande y de otras razas estudiadas. } \\
\hline Población & Lugar de colecta & $\begin{array}{c}\text { Altitud } \\
(\mathrm{m})\end{array}$ & $\begin{array}{l}\text { Latitud } \\
\text { Norte }\end{array}$ & $\begin{array}{c}\text { Longitud } \\
\text { Oeste }\end{array}$ \\
\hline 1 & Asunción Ixtaltepec, Oax. & 30 & $16^{\circ} 30^{\prime} 10^{\prime \prime}$ & $95^{\circ} 03^{\prime} 30^{\prime \prime}$ \\
\hline 2 & San Pedro Huamelula, Oax. & 77 & $16^{\circ} 01^{\prime} 30^{\prime}$, & $95^{\circ} 39^{\prime} 57^{\prime \prime}$ \\
\hline 4 & San Vicente Mazatán, Oax. & 70 & $16^{\circ} 07^{\prime} 20^{\prime \prime}$ & $95^{\circ} 25^{\prime} 25^{\prime}$, \\
\hline 6 & Santo Domingo Petapa, Oax. & 237 & $16^{\circ} 49^{\prime} 17^{\prime}$, & $95^{\circ} 08^{\prime} 20^{\prime \prime}$ \\
\hline 7 & San Dionisio del Mar, Oax. & 10 & $16^{\circ} 19^{\prime} 14^{\prime \prime}$ & $94^{\circ} 45^{\prime} 25^{\prime \prime}$ \\
\hline 8 & Santo Domingo Petapa, Oax. & 237 & $16^{\circ} 49^{\prime} 17^{\prime}$, & $95^{\circ} 08^{\prime} 20^{\prime \prime}$ \\
\hline 9 & Santo Domingo Petapa, Oax. & 237 & $16^{\circ} 49^{\prime} 17^{\prime \prime}$ & $95^{\circ} 08^{\prime} 20^{\prime \prime}$ \\
\hline 15 & San Pedro Huamelula, Oax. & 77 & $16^{\circ} 01^{\prime} 30^{\prime \prime}$ & $95^{\circ} 39^{\prime} 57^{\prime \prime}$ \\
\hline 16 & San Pedro Huamelula, Oax. & 77 & $16^{\circ} 01^{\prime} 30^{\prime \prime}$ & $95^{\circ} 39^{\prime} 57^{\prime \prime}$ \\
\hline 19 & Santiago Lachiguiri, Oax. & 1300 & $16^{\circ} 44^{\prime} 00^{\prime \prime}$ & $95^{\circ} 31^{\prime} 18^{\prime \prime}$ \\
\hline 20 & Santiago Lachiguiri, Oax. & 1100 & $16^{\circ} 40^{\prime} 20^{\prime}$, & $95^{\circ} 32^{\prime} 00^{\prime \prime}$ \\
\hline 23 & Zanatepec, Oax. & 89 & $16^{\circ} 28^{\prime} 35^{\prime}$, & $94^{\circ} 21^{\prime} 07^{\prime \prime}$ \\
\hline 24 & San Miguel Tenango, Oax. & 1550 & $16^{\circ} 16^{\prime} 00^{\prime \prime}$ & $95^{\circ} 36^{\prime} 00^{\prime \prime}$ \\
\hline 28 & Matías Romero, Oax. & 180 & $16^{\circ} 52^{\prime} 27^{\prime \prime}$ & $95^{\circ} 02^{\prime} 22^{\prime \prime}$ \\
\hline 31 & Guevea de Humboldt, Oax. & 500 & $16^{\circ} 38^{\prime} 49^{\prime \prime}$ & $95^{\circ} 30^{\prime} 33^{\prime \prime}$ \\
\hline 32 & San Juan Guichicovi, Oax. & 275 & $16^{\circ} 57^{\prime} 30^{\prime \prime}$ & $95^{\circ} 05^{\prime} 30^{\prime \prime}$ \\
\hline 33 & Santa Maria Guienagati, Oax. & 350 & $16^{\circ} 44^{\prime} 15^{\prime}$, & $95^{\circ} 21^{\prime} 14^{\prime \prime}$ \\
\hline CHIS-224 & San Felipe, Escuintla, Chis. & 100 & $15^{\circ} 20^{\prime}$ & $92^{\circ} 40^{\prime}$ \\
\hline CHIS-578 & Soyaló, Chis. & 800 & $16^{\circ} 54^{\prime}$ & $92^{\circ} 55^{\prime}$ \\
\hline GUER-96 & Petatlán, Gro. & 50 & $17^{\circ} 31^{\prime}$ & $101^{\circ} 17^{\prime}$ \\
\hline OAXA-54 & Niltepec, Oax. & 100 & $16^{\circ} 35^{\prime}$ & $94^{\circ} 36^{\prime}$ \\
\hline OAXA-244 & Costatitlán, Oax. & 250 & $16^{\circ} 32^{\prime}$ & $98^{\circ} 09^{\prime}$ \\
\hline VERA-39 & Gutiérrez Zamora, Ver. & 106 & $20^{\circ} 28^{\prime}$ & $97^{\circ} 05^{\prime}$ \\
\hline VERA-168 & Zapotalillo, Tihuatlán, Ver. & 140 & $20^{\circ} 37^{\prime}$ & $97^{\circ} 34^{\prime}$ \\
\hline YUCA-108 & Kantunil, Yuc. & 30 & $20^{\circ} 47^{\prime}$ & $89^{\circ} 01^{\prime}$ \\
\hline YUCA-117 & Hunucmá, Yuc. & 30 & $21^{\circ} 01^{\prime}$ & $89^{\circ} 54^{\prime}$ \\
\hline LADA-434 & Hobompich, QR. & 22 & $19^{\circ} 48^{\prime} 03^{\prime}$, & $88^{\circ} 21^{\prime}$ \\
\hline
\end{tabular}




\section{Análisis de la diversidad genética}

Con base en la lectura de los zimogramas se registró, en cada población, el número promedio de alelos por locus, las frecuencias génicas, el porcentaje de loci polimórficos, al considerar como tales aquéllos en los que la ocurrencia del alelo más frecuente no fuera superior a $95 \%$, la heterocigosidad esperada para cada subgrupo y la total $\left(\mathrm{H}_{\mathrm{s}} \mathrm{y} \mathrm{H}_{\mathrm{t}}\right)$, y el grado de diferenciación genética entre poblaciones $(\mathrm{Gst})$, calculado como: $\mathrm{Gst}=1-\left(\mathrm{H}_{\mathrm{s}} / \mathrm{H}_{\mathrm{t}}\right)$, donde $\mathrm{H}_{\mathrm{s}}$ es la heterocigosidad de los individuos en relación con el subgrupo al que pertencen y $\mathrm{H}_{\mathrm{t}}$ es la heterocigosidad calculada en relación con el total de poblaciones del estudio. Para la estimación de estos parámetros se utilizaron los programas POPGENE versión 1.31 (Yeh et al., 1999) y herramientas para análisis genéticos de poblaciones (Tools for Population Genetic Analyses, TFPGA) (Miller, 1997).

\section{Análisis de agrupamientos}

Con las frecuencias génicas se generó una matriz de distancias genéticas modificadas de Rogers mediante el paquete NTSYS-pc (Rohlf, 1993), y a partir de ésta se hizo un análisis de conglomerados con el método de Agrupamiento de Vecinos (Neighbor-Joining) (Saitou y Nei, 1987) mediante el programa análisis de datos genéticos (Genetic Data Analysis, GDA), versión 1.0 (Lewis y Zaykin, 2001); el filograma obtenido fue visualizado con el paquete TREEVIEW, versión 1.6.1 (Page, 1996).

\section{RESULTADOS Y DISCUSIÓN}

\section{Número de alelos}

Se encontraron 49 alelos distribuidos en las 40 poblaciones locales estudiadas, con un promedio de 2.4 alelos por locus para las poblaciones de Zapalote Chico (Cuadro 3) y de 2.6 para las de Maíz Grande (Cuadro 4), los cuales son valores que están dentro de los intervalos típicos para los estudios con isoenzimas en maíz y aportan un primer elemento para afirmar que los maíces del Istmo de Tehuantepec, representados por la raza Zapalote Chico y el complejo de Maíz Grande, tienen una variabilidad genética comparable con la de otros grupos raciales. Por ejemplo, Sánchez et al. (2000) encontraron variación en el número promedio de alelos desde 1.5 en la raza Harinoso de Ocho, hasta 3.4 en la raza Zamorano Amarillo; en tanto que Goodman y Stuber (1983) identificaron en las razas de Bolivia Paru con 1.3 y Yungueño con 2.7. Al comparar el número promedio de alelos por locus obtenido en esta investigación con los informados en otros estudios (Cuadro 5), se aprecian valores altos (9.3 y 6.4) en los estudios de Sánchez et al. (2000) y Bretting et al. (1990), mientras que Smith (1986) encontró un valor de 2.8, similar al obtenido en este trabajo. Al parecer, independientemente de las poblaciones, la multiplicidad de alelos es inherente al locus Glu1; lo cual coincide con los estudios realizados por Goodman y Stuber (1983), Smith (1986), Bretting et al. (1990) y Sánchez et al. (2000).

\section{Porcentaje de loci polimórficos}

Las 50 poblaciones estudiadas presentaron $69.5 \%$ de loci polimórficos, y algo menores, $58 \%$ para las poblaciones de Zapalote Chico (Cuadro 3) y $68 \%$ para las de Maíz Grande (Cuadro 4); para el mismo conjunto de loci, Sánchez et al., (2000) encontraron valores desde $38.8 \%$ para la raza Harinoso de Ocho, hasta $88.8 \%$ en las razas Tehua y Tablilla de Ocho; y Goodman y Stuber (1983) con razas de Bolivia identificaron en la raza Paru el menor porcentaje de loci polimórficos (33.3\%), y en las razas Yungueño y Argentino el mayor polimorfismo (77.7 \%). Estos resultados demuestran que la diversidad genética en los maíces de la región de estudio no es tan restringida como se suponía.

\section{Heterocigosidad}

La heterocigosidad esperada por locus en el grupo de poblaciones nativas y las representativas de otras razas fue igual (0.22) (Cuadro2), a pesar de que las 40 poblaciones nativas tuvieron un mayor promedio de alelos por locus; es probable que la mayor aparición de alelos raros (con frecuencia $<1 \%$ ), en el grupo de poblaciones nativas versus el grupo de poblaciones representativas (18 vs. 8 $\%$ de alelos raros), contribuyó a que la heterocigosidad fuera igual en ambos grupos. Al comparar los resultados de heterocigosidad esperada de los dos grupos con la obtenida en otros estudios (Cuadro 6), ésta fue similar a la obtenida por otros autores (Goodman y Stuber, 1983; Sánchez et al., 2000; Bretting et al., 1990 y Smith, 1986); lo que demuestra nuevamente que las poblaciones del Istmo de Tehuantepec tienen una diversidad comparable con la de otros grupos de poblaciones de maíz. Se ha postulado que las isoenzimas se ajustan a un modelo infinito de evolución, que junto con el hecho de ser marcadores esencialmente neutros a la selección (Kimura, 1983), genera nuevos alelos a tasas comparables entre poblaciones, con poca relación con las condiciones ambientales y sistemas agrícolas practicados. Lo anterior hace factible la inclusión de estos maíces en programas de fitomejoramiento, ya que poseen un cúmulo de complejos genéticos altamente favorables (Muñoz, 2005). 
Cuadro 3. Número de alelos por locus, número promedio de alelos (NPA), porcentaje de loci polimórficos (\% LP) y heterocigocidad esperada (Hs) en 23 poblaciones de maíz Zapalote Chico.

\begin{tabular}{|c|c|c|c|c|c|c|c|c|c|c|c|c|c|c|c|c|c|c|}
\hline \multirow[b]{2}{*}{ Población } & \multicolumn{15}{|c|}{ Locus } & \multirow[b]{2}{*}{$\mathrm{NPA}^{\dagger}$} & \multirow[b]{2}{*}{$\% \mathrm{LP}^{\dagger}$} & \multirow[b]{2}{*}{$\mathrm{Hs}^{\dagger}$} \\
\hline & Acpl & Acp4 & Cat3 & Est8 & Glu1 & Got1 & Idh1 & $I d h 2$ & $M d h 1$ & $M d h 2$ & $M d h 3$ & $M d h 4$ & $M d h 5$ & $P g d 1$ & Phil & & & \\
\hline 3 & 2 & 3 & 1 & 1 & 2 & 2 & 1 & 2 & 1 & 3 & 1 & 2 & 2 & 2 & 2 & 1.6 & 53 & 0.20 \\
\hline 5 & 2 & 2 & 2 & 1 & 4 & 2 & 1 & 2 & 1 & 3 & 1 & 1 & 2 & 2 & 1 & 1.6 & 47 & 0.19 \\
\hline 10 & 2 & 3 & 1 & 1 & 3 & 1 & 1 & 1 & 1 & 3 & 1 & 1 & 2 & 2 & 2 & 1.5 & 37 & 0.18 \\
\hline 11 & 2 & 3 & 1 & 2 & 2 & 2 & 1 & 2 & 1 & 2 & 1 & 1 & 2 & 2 & 2 & 1.6 & 53 & 0.17 \\
\hline 12 & 2 & 3 & 1 & 2 & 3 & 2 & 1 & 2 & 2 & 2 & 1 & 1 & 2 & 2 & 2 & 1.7 & 58 & 0.19 \\
\hline 13 & 2 & 2 & 1 & 1 & 2 & 2 & 1 & 2 & 1 & 2 & 1 & 1 & 1 & 2 & 4 & 1.5 & 42 & 0.17 \\
\hline 14 & 2 & 3 & 1 & 2 & 4 & 2 & 1 & 2 & 1 & 2 & 1 & 1 & 2 & 2 & 3 & 1.7 & 53 & 0.20 \\
\hline 17 & 2 & 3 & 1 & 3 & 3 & 2 & 1 & 2 & 2 & 2 & 1 & 1 & 2 & 2 & 2 & 1.7 & 58 & 0.21 \\
\hline 18 & 2 & 3 & 1 & 1 & 3 & 2 & 1 & 2 & 2 & 2 & 1 & 1 & 2 & 2 & 2 & 1.6 & 53 & 0.18 \\
\hline 21 & 2 & 3 & 1 & 3 & 3 & 1 & 1 & 2 & 1 & 2 & 1 & 1 & 2 & 2 & 2 & 1.6 & 47 & 0.21 \\
\hline 22 & 2 & 3 & 1 & 1 & 2 & 2 & 2 & 2 & 1 & 3 & 1 & 1 & 2 & 2 & 2 & 1.6 & 53 & 0.19 \\
\hline 25 & 2 & 3 & 1 & 2 & 3 & 2 & 1 & 2 & 2 & 2 & 1 & 1 & 2 & 2 & 2 & 1.7 & 58 & 0.21 \\
\hline 26 & 2 & 2 & 1 & 2 & 3 & 2 & 1 & 1 & 1 & 3 & 1 & 1 & 1 & 2 & 2 & 1.5 & 42 & 0.14 \\
\hline 27 & 2 & 3 & 1 & 1 & 2 & 1 & 1 & 2 & 1 & 2 & 1 & 2 & 2 & 2 & 2 & 1.5 & 47 & 0.18 \\
\hline 29 & 2 & 3 & 1 & 3 & 2 & 2 & 1 & 2 & 1 & 3 & 1 & 1 & 2 & 2 & 2 & 1.7 & 53 & 0.18 \\
\hline 30 & 2 & 3 & 1 & 1 & 2 & 3 & 1 & 2 & 2 & 2 & 1 & 2 & 2 & 2 & 2 & 1.7 & 58 & 0.21 \\
\hline 35 & 2 & 2 & 1 & 1 & 3 & 2 & 1 & 2 & 2 & 2 & 1 & 1 & 2 & 2 & 2 & 1.6 & 53 & 0.19 \\
\hline 36 & 2 & 2 & 1 & 1 & 2 & 1 & 1 & 2 & 2 & 2 & 1 & 1 & 2 & 2 & 2 & 1.5 & 47 & 0.16 \\
\hline 37 & 2 & 3 & 1 & 4 & 3 & 2 & 1 & 2 & 1 & 2 & 1 & 1 & 2 & 2 & 3 & 1.8 & 53 & 0.23 \\
\hline 38 & 2 & 3 & 1 & 1 & 2 & 3 & 1 & 2 & 1 & 2 & 1 & 1 & 2 & 2 & 2 & 1.6 & 47 & 0.20 \\
\hline 39 & 2 & 2 & 1 & 2 & 2 & 2 & 1 & 2 & 2 & 3 & 1 & 1 & 2 & 2 & 2 & 1.6 & 58 & 0.21 \\
\hline 40 & 2 & 2 & 1 & 1 & 2 & 2 & 1 & 2 & 1 & 2 & 1 & 1 & 2 & 2 & 1 & 1.4 & 42 & 0.14 \\
\hline Subtotal & 2 & 4 & 2 & 4 & 6 & 2 & 3 & 2 & 3 & 3 & 1 & 2 & 2 & 2 & 4 & 2.4 & 58 & 0.21 \\
\hline
\end{tabular}


Cuadro 4. Número de alelos por locus, número promedio de alelos (NPA), porcentaje de loci polimórficos (\% LP) y heterocigocidad esperada (Hs) en 17 poblaciones de Maíz Grande y 10 poblaciones representativas de otras razas de maíz.

\begin{tabular}{|c|c|c|c|c|c|c|c|c|c|c|c|c|c|c|c|c|c|c|}
\hline \multirow[b]{2}{*}{ Población } & \multicolumn{15}{|c|}{ Locus } & \multirow[b]{2}{*}{$\mathrm{NPA}^{\dagger}$} & \multirow[b]{2}{*}{$\% \mathrm{LP}^{\dagger}$} & \multirow[b]{2}{*}{$\mathrm{Hs}^{\dagger}$} \\
\hline & Acp1 & Acp 4 & Cat 3 & Est 8 & Glu1 & Got1 & Idhl & $I d h 2$ & Mdh1 & Mdh2 & $M d h 3$ & Mdh4 & $M d h 5$ & $P g d 1$ & Phil & & & \\
\hline 1 & 2 & 3 & 1 & 1 & 2 & 2 & 2 & 2 & 1 & 3 & 1 & 1 & 2 & 2 & 2 & 1.6 & 53 & 0.20 \\
\hline 2 & 2 & 4 & 1 & 1 & 2 & 1 & 1 & 2 & 2 & 2 & 1 & 2 & 2 & 2 & 2 & 1.6 & 53 & 0.21 \\
\hline 4 & 2 & 2 & 1 & 1 & 2 & 2 & 1 & 2 & 1 & 2 & 1 & 1 & 2 & 2 & 2 & 1.5 & 47 & 0.20 \\
\hline 6 & 2 & 4 & 1 & 2 & 3 & 2 & 1 & 2 & 1 & 2 & 1 & 1 & 2 & 2 & 2 & 1.7 & 53 & 0.22 \\
\hline 7 & 2 & 4 & 1 & 2 & 4 & 2 & 1 & 2 & 1 & 3 & 1 & 2 & 2 & 2 & 2 & 1.8 & 58 & 0.24 \\
\hline 8 & 2 & 2 & 1 & 2 & 3 & 2 & 1 & 2 & 1 & 2 & 1 & 2 & 2 & 2 & 1 & 1.6 & 53 & 0.21 \\
\hline 9 & 2 & 3 & 1 & 1 & 2 & 2 & 1 & 2 & 2 & 2 & 1 & 2 & 2 & 2 & 3 & 1.7 & 58 & 0.20 \\
\hline 15 & 2 & 3 & 1 & 1 & 2 & 1 & 2 & 2 & 2 & 2 & 1 & 1 & 1 & 2 & 2 & 1.5 & 47 & 0.14 \\
\hline 16 & 2 & 3 & 1 & 1 & 3 & 2 & 1 & 1 & 2 & 2 & 1 & 1 & 2 & 1 & 2 & 1.5 & 42 & 0.15 \\
\hline 19 & 2 & 3 & 1 & 1 & 3 & 2 & 2 & 2 & 2 & 2 & 1 & 2 & 2 & 1 & 2 & 1.7 & 58 & 0.19 \\
\hline 20 & 2 & 3 & 1 & 2 & 3 & 1 & 1 & 2 & 2 & 2 & 1 & 1 & 1 & 2 & 3 & 1.6 & 47 & 0.17 \\
\hline 23 & 2 & 3 & 2 & 2 & 2 & 1 & 1 & 2 & 2 & 3 & 1 & 1 & 2 & 2 & 1 & 1.6 & 53 & 0.20 \\
\hline 24 & 2 & 3 & 1 & 1 & 2 & 2 & 1 & 2 & 1 & 3 & 1 & 2 & 2 & 2 & 1 & 1.6 & 47 & 0.20 \\
\hline 28 & 2 & 3 & 1 & 2 & 3 & 2 & 1 & 2 & 2 & 2 & 1 & 1 & 2 & 2 & 2 & 1.7 & 58 & 0.18 \\
\hline 31 & 2 & 3 & 1 & 2 & 3 & 2 & 2 & 2 & 2 & 2 & 1 & 2 & 1 & 2 & 2 & 1.7 & 63 & 0.21 \\
\hline 32 & 2 & 3 & 1 & 2 & 2 & 2 & 1 & 2 & 2 & 2 & 1 & 1 & 2 & 2 & 2 & 1.6 & 58 & 0.22 \\
\hline 33 & 2 & 2 & 1 & 1 & 3 & 1 & 1 & 2 & 2 & 2 & 1 & 1 & 2 & 2 & 2 & 1.5 & 47 & 0.17 \\
\hline Subtotal & 2 & 5 & 2 & 4 & 6 & 3 & 3 & 2 & 2 & 3 & 1 & 2 & 2 & 2 & 4 & 2.6 & 68 & 0.22 \\
\hline CHIS-224 & 2 & 3 & 1 & 1 & 3 & 1 & 1 & 2 & 1 & 2 & 1 & 1 & 2 & 2 & 1 & 1.5 & 37 & 0.17 \\
\hline CHIS-578 & 2 & 3 & 1 & 1 & 3 & 2 & 1 & 2 & 1 & 2 & 1 & 2 & 2 & 2 & 1 & 1.6 & 47 & 0.17 \\
\hline GUER-96 & 2 & 3 & 1 & 1 & 2 & 1 & 1 & 2 & 2 & 2 & 1 & 2 & 2 & 2 & 1 & 1.5 & 47 & 0.21 \\
\hline OAXA-54 & 2 & 3 & 1 & 1 & 3 & 2 & 1 & 2 & 1 & 2 & 1 & 1 & 2 & 2 & 1 & 1.5 & 42 & 0.18 \\
\hline OAXA-244 & 2 & 3 & 1 & 1 & 3 & 1 & 2 & 2 & 2 & 2 & 1 & 2 & 2 & 2 & 1 & 1.6 & 53 & 0.17 \\
\hline VERA-39 & 2 & 3 & 1 & 1 & 3 & 2 & 1 & 2 & 2 & 2 & 2 & 2 & 2 & 1 & 1 & 1.6 & 53 & 0.22 \\
\hline VERA-168 & 2 & 2 & 2 & 1 & 2 & 1 & 2 & 2 & 1 & 2 & 1 & 1 & 2 & 2 & 1 & 1.5 & 47 & 0.16 \\
\hline YUCA-108 & 2 & 2 & 1 & 1 & 2 & 1 & 1 & 2 & 1 & 2 & 1 & 1 & 2 & 2 & 2 & 1.4 & 42 & 0.14 \\
\hline YUCA-117 & 3 & 3 & 1 & 1 & 2 & 2 & 1 & 1 & 1 & 2 & 1 & 1 & 2 & 2 & 1 & 1.5 & 37 & 0.17 \\
\hline LADA-434 & 2 & 3 & 1 & 1 & 2 & 2 & 1 & 1 & 1 & 1 & 1 & 1 & 2 & 2 & 1 & 1.4 & 32 & 0.13 \\
\hline Subtotal & 3 & 3 & 2 & 1 & 5 & 2 & 3 & 2 & 2 & 2 & 2 & 2 & 2 & 2 & 2 & 2.1 & 74 & 0.22 \\
\hline
\end{tabular}


Cuadro 5. Comparación del número de alelos entre germoplasma de diferentes estudios con el mismo grupo de loci de isoenzimas usado en las poblaciones del Istmo de Tehuantepec y las 10 razas típicas de maíz.

\begin{tabular}{|c|c|c|c|c|c|c|c|c|c|c|c|c|c|c|c|c|c|c|c|c|c|}
\hline \multirow[b]{2}{*}{ Germoplasma } & \multicolumn{19}{|c|}{ Número de alelos en cada locus } & \multirow[b]{2}{*}{ APL } & \multirow[b]{2}{*}{ PLP 95\% } \\
\hline & $\bar{s}$ & $\stackrel{*}{*}$ & $\underset{\mathbb{Z}}{\mathbb{7}}$ & 3 & 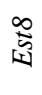 & త্ত & $\overline{0}$ & $\widetilde{\Xi}$ & 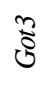 & 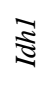 & $\underset{\Xi}{\Xi}$ & 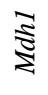 & $\underset{\Xi}{\mathbb{Z}}$ & $\stackrel{2}{\mathbb{Z}}$ & $\underset{\mathbb{Z}}{\mathbb{Z}}$ & 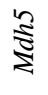 & $\begin{array}{l}\bar{\Xi} \\
2 \\
2\end{array}$ & $\begin{array}{l}\mathbb{Z} \\
\mathbb{2} \\
2\end{array}$ & $\underset{\mathbb{Z}}{\bar{Z}}$ & & \\
\hline 40 Pob. Istmo & 2 & 5 & 1 & 2 & 4 & 7 & 3 & 1 & 1 & 3 & 2 & 3 & 3 & 1 & 2 & 2 & 2 & 1 & 4 & 2.6 & 68.4 \\
\hline 10 Pob. Repr. ${ }^{\Upsilon 1}$ & 3 & 3 & 1 & 2 & 1 & 5 & 2 & 1 & 1 & 3 & 2 & 2 & 2 & 2 & 2 & 2 & 2 & 1 & 2 & 2.1 & 73.6 \\
\hline 31 Razas Bolivia ${ }^{\xi}$ & 6 & - & 4 & 8 & 6 & 16 & 4 & 3 & 2 & 4 & 5 & 7 & 5 & 4 & 3 & 6 & 5 & 5 & 6 & 5.5 & 88.9 \\
\hline 59 Razas Méx. ${ }^{\xi \xi}$ & 10 & - & 5 & 15 & 9 & 28 & 8 & 5 & 6 & 6 & 10 & 10 & 10 & 5 & 13 & 6 & 9 & 5 & 8 & 9.3 & 72.2 \\
\hline 19 Razas Guat. ${ }^{\phi}$ & 9 & - & 4 & 8 & 5 & 17 & 7 & 5 & 6 & 6 & 4 & 7 & 9 & 5 & 4 & 5 & 6 & 3 & 6 & 6.4 & 88.9 \\
\hline 47 Pob. USA ${ }^{\phi \phi}$ & 5 & - & 2 & 2 & - & 7 & 2 & 2 & 1 & 2 & 3 & 3 & 5 & 2 & 1 & 2 & 2 & 3 & 3 & 2.8 & 82.4 \\
\hline
\end{tabular}

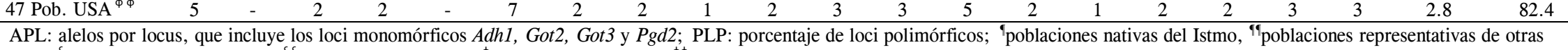
razas, ${ }_{\xi}^{\xi}$ Goodman y Stuber (1983), ${ }^{\xi \xi}$ Sánchez et al. (2000), ${ }^{\phi}$ Bretting et al. $(1990),{ }^{\phi \phi}$ Smith (1986).

Cuadro 6. Heterocigocidad esperada de 40 poblaciones de maíz del Istmo de Tehuantepec y poblaciones representativas de otras razas típicas de maíz.

Heterocigocidad esperada para cada locus

\begin{tabular}{|c|c|c|c|c|c|c|c|c|c|c|c|c|c|c|c|c|c|c|c|c|}
\hline Germoplasma & $\frac{\bar{s}}{\pi}$ & $\stackrel{ \pm}{*}$ & $\underset{\mathbf{z}}{\mathbb{7}}$ & $\tilde{z}$ & 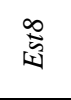 & క & $\begin{array}{c}\overline{5} \\
\end{array}$ & $\stackrel{\widetilde{\Xi}}{8}$ & $\underset{0}{0}$ & ミ & 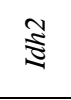 & $\stackrel{\Xi}{\mathbb{Z}}$ & $\stackrel{\Xi}{\Xi}$ & $\stackrel{?}{\xi}$ & $\underset{\Xi}{\mathbb{Z}}$ & $\stackrel{2}{z}$ & $\begin{array}{l}\bar{D} \\
\mathbb{\infty}\end{array}$ & $\begin{array}{l}\widetilde{D} \\
\mathbb{\infty} \\
\mathbb{2}^{\circ}\end{array}$ & 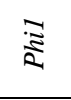 & Media \\
\hline 40 Pob. Istmo ${ }^{\dagger}$ & 0.50 & 0.63 & 0.0 & 0.01 & 0.13 & 0.55 & 0.19 & 0.00 & 0.00 & 0.04 & 0.34 & 0.11 & 0.51 & 0.00 & 0.03 & 0.36 & 0.50 & 0.00 & 0.25 & 0.22 \\
\hline 10 Pob. Repr. ${ }^{\dagger \dagger}$ & 0.54 & 0.65 & 0.0 & 0.02 & 0.00 & 0.6 & 0.24 & 0.00 & 0.00 & 0.1 & 0.29 & 0.17 & 0.46 & 0.04 & 0.17 & 0.46 & 0.50 & 0.00 & 0.02 & 0.22 \\
\hline 31 Razas Bol. & 0.51 & - & 0.06 & 0.42 & 0.15 & 0.48 & 0.44 & 0.04 & 0.01 & 0.13 & 0.14 & 0.21 & 0.49 & 0.09 & 0.01 & 0.36 & 0.52 & 0.04 & 0.12 & 0.23 \\
\hline 59 Razas Méx. 9 & 0.63 & - & 0.02 & 0.2 & 0.19 & 0.83 & 0.22 & 0.01 & 0.05 & 0.32 & 0.47 & 0.19 & 0.63 & 0.18 & 0.04 & 0.28 & 0.45 & 0.01 & 0.22 & 0.27 \\
\hline 19 Razas Guat. "ף & 0.60 & - & 0.05 & 0.09 & 0.34 & 0.82 & 0.23 & 0.01 & 0.04 & 0.45 & 0.46 & 0.15 & 0.52 & 0.13 & 0.02 & 0.24 & 0.37 & 0.00 & 0.28 & 0.27 \\
\hline 47 Pob. USA ${ }^{\xi}$ & 0.60 & - & 0.29 & 0.22 & - & 0.64 & 0.04 & 0.14 & 0.00 & 0.03 & 0.5 & 0.22 & 0.67 & 0.18 & 0.00 & 0.06 & 0.43 & 0.02 & 0.21 & 0.25 \\
\hline
\end{tabular}




\section{Frecuencias génicas}

El número de alelos raros, con frecuencia $<0.01$, fue de $12(23 \%)$ en las 50 poblaciones estudiadas. Para este tipo de alelos, diversos autores han reportado valores semejantes a los aquí obtenidos. Por ejemplo, Smith (1986) reportó nueve de 56 alelos (16\%) en 47 poblaciones de polinización libre del complejo racial dentado de la faja maicera; Revilla y Tracy (1995) encontraron 13 de 60 alelos $(21 \%)$ en poblaciones de maíz dulce y un caso extremo es el informado por Sánchez et al. (2000) para 59 razas de México, en las cuales de 303 alelos observados, 194 (64 \%) tuvieron frecuencias bajas. Es poco conocido el efecto fenotípico asociado con las frecuencias altas de algunos alelos; sin embargo, las diferencias entre frecuencias génicas del germoplasma es un buen indicativo de su contribución valiosa a los programas de mejoramiento (Lu et al., 2002).

La experiencia de décadas en fitomejoramiento muestra que las poblaciones de maíz provenientes de diferentes regiones presentan cierta divergencia en sus frecuencias génicas, debido posiblemente a uno o a la combinación de varios factores como deriva genética, mutación, cambios en la presión de selección asociados con desplazamientos a nuevos ambientes y aislamiento reproductivo (Doebley et al., 1986). Ello ha originado que existan poblaciones con combinaciones únicas de alelos de isoenzimas, y permitido su ubicación dentro de un grupo particular de poblaciones o dentro de una raza. Para las poblaciones de maíz del Istmo de Tehuantepec, Oaxaca, los resultados indican que han acumulado alelos en diferentes frecuencias génicas que las distinguen de las poblaciones geográficamente cercanas, como las razas de Guatemala (Bretting et al., 1990). Sin duda, la selección natural y los factores ambientales adversos como calor, sequía, viento y plagas (Muñoz, 2005), aunado a la selección artificial para obtener derivados alimenticios de calidad culinaria, han conducido a las poblaciones de maíz del Istmo de Tehuantepec por una ruta evolutiva sustancialmente diferente a la de otras poblaciones a lo largo de 5000 años (Gutiérrez-Nava et al., 1998).

\section{Diferenciación genética entre poblaciones}

La diferenciación genética relativa (Gst) es un parámetro introducido por Nei (1975) como una extensión de $\mathrm{FsT}^{1}$ para el caso de alelos múltiples, comunes en

\footnotetext{
${ }^{1}$ Fst es el grado de diferenciación genética de las poblaciones, calculado con información de marcadores dominantes o codominantes con uno o dos alelos por locus. Valores bajos de Fst indican cercanía genética entre poblaciones, y valores altos indican fijación de diferentes alelos, debido a un reducido flujo génico entre poblaciones a causa de aislamiento, deriva genética o cambios en la presión de selección.
}

isoenzimas, y de manera práctica denota la proporción de la variabilidad entre poblaciones, y es el complemento atribuido a la variabilidad dentro de las mismas. En el Cuadro 7 se muestra un valor de Gst de 0.126 en las poblaciones del Istmo; es decir, alrededor de $88 \%$ de la variación isoenzimática reside dentro de poblaciones y 12 $\%$ entre ellas, con mayor diferenciación genética relativa entre las poblaciones representativas de otras razas, atribuible a sus diferentes orígenes y patrimonios genéticos.

Con base en el valor de Gst y en el criterio establecido por Snyder et al. (1985), que considera que una divergencia genética moderada posee valores de Gsт de 0.05 a 0.15 , y alta de 0.15 a 0.25 , las poblaciones del Istmo de Tehuantepec se clasifican con un nivel moderado de divergencia genética. En otros estudios que se consignan en el Cuadro 7 se obtuvieron valores similares de Gst al de los maíces del Istmo de Tehuantepec, como en las poblaciones de maíz del norte de España (Llauradó et al., 1993). El valor bajo de Gst observado en las poblaciones nativas puede atribuirse a que el área explorada fue relativamente pequeña y con predominancia de la raza Zapalote Chico; a diferencia de Doebley et al. (1985) y Pflüger y Schlatter (1996), quienes con muestras representativas a nivel de país, y Lu et al. (2002), que evaluaron 2000 poblaciones colectadas en el suroeste de China, encontraron valores altos de Gst. Llauradó et al. (1993) obtuvieron un valor de Gst similar al encontrado en este estudio probablemente porque las 86 poblaciones nativas evaluadas provinieron de una sola región del norte de España.

\section{Análisis de agrupamiento}

Los valores de las relaciones de similitud separaron a las 50 poblaciones en dos grupos. El grupo basal, indicado con el número II, lo constituyeron las poblaciones de la raza Zapalote Chico, mientras que en el grupo superior, indicado con el número I, predominaron las poblaciones de áreas de mayor altitud y más húmedas de la región, correspondiente al Maíz Gande, con influencia de la raza Tuxpeño y alguna similitud con otras razas como Vandeño y Tepecintle. El Grupo I se caracterizó por la frecuencia alta de los alelos Glu1-2, Pgd1-2 e Idh2$6(0.48,0.60$ y 0.30 , respectivamente), mientras que el Grupo II se caracterizó por frecuencias altas de los alelos Glu1-6, Pdg1-3.8 e Idh2-4 (0.67, 0.56 y 0.84 , respectivamente).

La variación de las frecuencias génicas entre los grupos detectados, no fue tan marcada como la obtenida para las colecciones de maíces cristalinos y dentados de los Estados Unidos (Doebley et al., 1986), para los que se reportaron al menos 12 alelos con fuerte discontinuidad; tal discontinuidad puede estar implicada en la alta 
heterosis mostrada por los híbridos entre estas poblaciones y su consistente respuesta a la selección. El número y tipo de loci que más discrimina a las poblaciones es varia- ble. Por ejemplo, Pflüger y Schlatter (1996) encontraron que los loci Acp1, Adh1, Cat3, E8 y Mdh2, fueron los más discriminantes para 40 poblaciones de maíz

Cuadro 7. Valores de la diferenciación genética relativa (Gst) de las poblaciones nativas, representativas de otras razas, total y de otros estudios para 19 loci de maíz evaluados.

\begin{tabular}{|c|c|c|c|c|c|c|c|}
\hline Locus & Nativas $^{\dagger}$ & Típicas $^{\dagger}$ & Global $^{\dagger}$ & 34 razas México $^{\dagger \dagger}$ & 18 razas Argentina ${ }^{\dagger \dagger \dagger}$ & 27 pob. China.' & 86 pob. España" \\
\hline Acpl & 0.078 & 0.279 & 0.122 & 0.269 & 0.212 & 0.233 & - \\
\hline Acp 4 & 0.122 & 0.154 & 0.132 & - & 0.206 & 0.338 & - \\
\hline Adh1 & - & - & - & 0.321 & 0.492 & 0.182 & 0.085 \\
\hline Cat3 & 0.083 & 0.091 & 0.090 & 0.338 & 0.224 & 0.159 & - \\
\hline Est8 & 0.139 & - & 0.148 & 0.203 & 0.148 & 0.034 & 0.127 \\
\hline Glu1 & 0.163 & 0.222 & 0.189 & 0.278 & - & - & 0.109 \\
\hline Got1 & 0.092 & 0.348 & 0.154 & 0.250 & - & 0.400 & 0.118 \\
\hline Got 2 & - & - & - & 0.263 & - & 0.100 & 0.109 \\
\hline Got3 & - & - & - & 0.200 & - & - & - \\
\hline Idh1 & 0.375 & 0.356 & 0.371 & 0.233 & - & - & 0.102 \\
\hline$I d h 2$ & 0.136 & 0.185 & 0.146 & 0.283 & - & - & 0.116 \\
\hline$M d h 1$ & 0.107 & 0.375 & 0.186 & 0.342 & 0.381 & 0.383 & 0.323 \\
\hline$M d h 2$ & 0.093 & 0.253 & 0.124 & 0.256 & 0.167 & 0.233 & 0.118 \\
\hline$M d h 3$ & - & 0.184 & 0.200 & 0.362 & 0.065 & 0.364 & 0.243 \\
\hline Mdh4 & 0.059 & 0.288 & 0.216 & 0.356 & 0.107 & - & - \\
\hline Mdh5 & 0.122 & 0.152 & 0.139 & 0.361 & 0.086 & - & 0.103 \\
\hline Pgd1 & 0.174 & 0.271 & 0.194 & 0.273 & - & 0.424 & 0.140 \\
\hline $\operatorname{Pgd} 2$ & - & - & - & 0.240 & - & 0.469 & 0.643 \\
\hline Phil & 0.105 & 0.091 & 0.126 & 0.215 & 0.207 & - & 0.259 \\
\hline Gst TOTAL & 0.126 & 0.235 & 0.154 & 0.276 & 0.218 & 0.281 & 0.128 \\
\hline
\end{tabular}

†Valores obtenidos en este trabajo; ${ }^{\dagger \dagger}$ Doebley et al. (1985); ${ }^{\dagger \dagger}$ Pflüger y Schlatter (1996); 'Lu et al. (2002); "Llauradó et al. (1993).

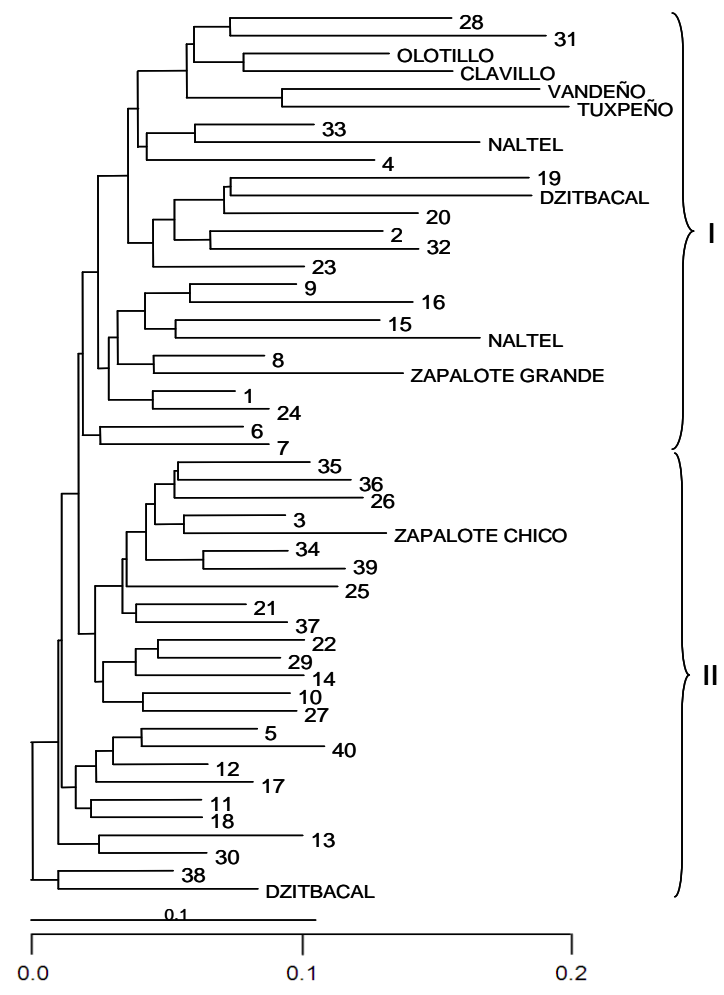

Distancia modificada de Rogers

Figura 1. Filograma del análisis de agrupamiento para 50 poblaciones de maíz obtenidos con la matriz de distancias modificadas de Rogers derivadas de 52 alelos de isoenzimas y el método de Agrupamiento de Vecinos (Neighbor-Joining). Los números arábigos indican el número de población. 


\section{CONCLUSIONES}

En la región explorada del Istmo de Tehuantepec las poblaciones nativas tienen una proporción relativamente alta de alelos raros $(23 \%)$, valores bajos para el número de alelos por locus (2.6) y porcentaje de alelos polimórficos (68.4), y heterocigosidad (0.22) comparable con otras poblaciones estudiadas.

La diferenciación genética de las poblaciones de maíz del Isto de Tehuantepec fue moderada, con una variabilidad de 88 y $12 \%$ dentro y entre poblaciones, respectivamente.

Se identificaron dos grupos de diversidad genética cuyas frecuencias génicas tuvieron correspondencia con una caracterización morfológica realizada con anterioridad, que permite separar las poblaciones de Maíz Grande de las de Zapalote Chico.

\section{BIBLIOGRAFÍA}

Bretting P K, M M Goodman, C W Stuber (1990) Isozymatic variation in Guatemalan races of maize. Amer. J. Bot. 77:211-225.

DBMU (1992) Data of the Latin American Maize Project. Also Maize Data from Agriculture Canada, CIMMYT, Central and South American Countries and USDA Agricultural Research Service. Compact Disc. GRIN Database Management Unit. USDA-ARS-PSI-NGRL. Beltsville, MA.

Doebley J F, M M Goodman, C W Stuber (1985) Isozyme variation in races of maize from Mexico. Amer. J. Bot. 72:629-639.

Doebley J F, M M Goodman, C W Stuber (1986) Exceptional genetic divergence of Northern flint corn. Amer. J. Bot. 73:64-69.

Goodman M M, C W Stuber (1983) Races of maize. VI. Isozyme variation among races of maize in Bolivia. Maydica 28:169187.

Gutiérrez-Nava M L, C A Warren, P León, V Walbot (1998) Transcriptionally active MuDR, the regulatory element of the mutator transposable element family of Zea mays, is present in some accessions of the Mexican land race Zapalote Chico. Genetics 149:329-346.

Kimura M (1983) The Neutral Theory of Molecular Evolution. Cambridge University Press. London. 384 p.

Lewis P O, D Zaykin (2001) Genetic Data Analysis: computer program for the analysis of allelic data. Version 1.0 (d16c). Disponible en: http://lewis.eeb.uconn.edu/lewishome/software.html; (Junio 2008).

Llauradó M, J Moreno G, P Arús (1993) Classification of northern Spanish populations of maize by methods of numerical taxonomy. II. Isozyme variation. Maydica 38:249-258.
López R G, A Santacruz V, A Muñoz O, F Castillo G, L Córdova T, H Vaquera H (2005) Caracterización morfológica de poblaciones nativas de maíz del Istmo de Tehuantepec, Oaxaca. Interciencia 30:284-290.

Lu H, J S Li, J L Liu, R Bernardo (2002) Allozyme polymorphisms of maize populations from southwestern China. Theor. Appl. Genet. 104:119-126.

Miller M P (1997) Tools for population genetic analyses (TFPGA) 1.3: A Windows program for the analysis of allozyme and molecular population genetic data. Disponible en: http://www.marksgeneticsoftware.net/tfpga.htm (Febrero 2010).

Muñoz O A (2005) Centli-maíz. Prehistoria e Historia, Diversidad, Potencial, Origen Genético y Geográfico. $2^{\mathrm{a}}$. ed. Colegio de Postgraduados. Montecillo, Texcoco, Edo. de México. 210 p.

Nei M (1975) Molecular Population Genetics and Evolution. North Holland/American Elsevier. Amsterdam. 288 p.

Page R D M (1996) Treeview: An application to display phylogenetic trees on personal computers. Comput. Appl. Biosci. 12:357358.

Pflüger L A, A R Schlatter (1996) Isozyme variation in some races of maize from Argentina. Genet. Res. Crop Evol. 43:357-362.

Ramírez F A, H H Ángeles A, J D Molina G (1988) Selección familial de progenies autofecundadas en una población de maíz (Zea mays L.) de la raza Zapalote Chico. Agrociencia 74:103-114.

Revilla P, W F Tracy (1995) Isozyme variation and phylogetic relationships among open-pollinated sweet corn cultivars. Crop Sci. 35:219-227.

Rohlf F J (1993) NTSYS-pc Numerical Taxonomy and Multivariate Analysis System. Versión 1.8. Department of Ecology and Evolution. State University of New York. New York, N.Y.

Saitou N, M Nei (1987) The neighbor-joining method: a new method for reconstructing phylogenetic trees. Mol. Biol. Evol. 4:406425 .

Sánchez G J J, M M Goodman, C W Stuber (2000). Isozymatic and morphological diversity in the races of maize of Mexico. Econ. Bot. 54:43-59.

SIAP (2009) Anuario Estadístico de la Producción Agrícola. Estado de Oaxaca. Servicio de Información Agroalimentaria y Pesquera. SAGARPA. México, D.F. Disponible en: http://www.siap.gob.mx/aagricola_siap/icultivo/index.jsp (Noviembre 2009).

Smith J S C (1986) Genetic diversity whitin the corn belt dent racial complex of maize (Zea mays L.). Maydica 31:349-367.

Snyder L A, D Freifelder, D L Hartl (1985) General Genetics. Jones \& Bartlett. Boston, MA. $666 \mathrm{p}$

Stuber C W, J F Wendel, M M Goodman, J S C Smith (1988) Techniques and scoring procedures for starch gel electrophoresis of enzymes from maize (Zea mays L.). North Carolina Agric. Res. Ser. Tech. Bull. 286. North Carolina State University. Raleigh, NC. 87 p.

Yeh C F, R Yang, T Boyle (1999) POPGENE Version 1.31. Microsoft Windows-based Freeware for Population Genetic Analysis. Quik User Guide. University of Alberta and Center for International Forestry Research. Edmonton, AB. Canada. 29 p. 